\title{
Vascular endothelial growth factor mRNA expression in minimal change, membranous, and diabetic nephropathy demonstrated by non-isotopic in situ hybridisation
}

\author{
E Bailey, M J Bottomley, S Westwell, J H Pringle, P N Furness, J Feehally,
} P E C Brenchley, S J Harper

\begin{abstract}
Aim-To investigate vascular endothelial growth factor (VEGF) mRNA expression in glomerular disease in the context of heavy proteinuria.

Methods-Non-radioisotopic in situ hybridisation was performed using a cocktail of 12 deoxyoligonucleotides complementary to VEGF mRNA labelled during solid phase synthesis with 2,4dinitrophenyl. Archival renal biopsies were studied from cases of minimal change nephropathy, membranous nephropathy, diabetic nephropathy, and controls, matched for age, sex, race, and storage time. Hybrid detection used NBT/ BCIP colorimetric development.

Results-More VEGF mRNA positive glomerular cells per unit cross sectional diameter were seen in minimal change nephropathy (mean (SEM), 19.35 (1.5)) compared with controls (12.6 (1.73)), $\mathbf{p}<0.01$. In contrast, fewer were seen in diabetic nephropathy $(5.93(0.97))$ compared with controls $(9.97(1.25)), \mathrm{p}<0.03$. Analysis of membranous nephropathy (10 (1.62)) showed no difference from controls (10.98 (1.51)), NS. In addition, in minimal change nephropathy there was a significant correlation between 24 hour protein excretion at the time of biopsy and the number of VEGF mRNA cells per glomerulus $(r=0.08, p=0.01)$.

Conclusions-Using non-radioisotopic in situ hybridisation, VEGF mRNA is almost exclusively expressed by visceral glomerular epithelial cells. Abnormal numbers of cells are seen in both minimal change and diabetic nephropathy. As VEGF exists in a number of functionally distinct isoforms, further study of qualitative VEGF isoform expression in diagnostic groups is indicated.

(f Clin Pathol 1999;52:735-738)
\end{abstract}

Keywords: vascular permeability factor; vascular endothelial growth factor; proteinuria; glomerulonephritis

Hospital,

Westbury-on-Trym,

Bristol BS10 5NB, UK

$S$ Westwell

S J Harper

Correspondence to:

Dr Harper

Accepted for publication 21 April 1999 ria is clinically important not only because of the nephrotic syndrome but also because it influences the progression of established glomerular lesions. ${ }^{1}$ The pathophysiology of proteinuria is poorly understood but one factor which has been implicated is vascular endothelial growth factor (VEGF).

VEGF, also known as vascular permeability factor (VPF), is a dimeric glycoprotein which mediates increased vascular permeability and endothelial mitogenesis. Radioisotopic in situ hybridisation has shown that the production site is primarily the visceral glomerular epithelial cell, ${ }^{23}$ while VEGF receptors are believed to be expressed by glomerular endothelial cells. ${ }^{4}$

The intimate anatomical juxtaposition of the source of the VEGF and its receptors, ${ }^{2}$ its extreme potency as a mediator of vascular permeability, ${ }^{5}$ the ability of some forms of VEGF to bind to heparan sulphate (an important contributor the anionic nature of the glomerular basement membrane), and the ability of VEGF to induce endothelial fenestrations $^{6}$ have together suggested that VEGF is implicated in the control of normal glomerular permselectivity in health and in the development of proteinuria in glomerular disease. $^{7}$ However, our knowledge of its expression in diagnostic groups is limited. Such investigation has been hindered by the lack of good commercially available probes and antibodies, the sparcity of tissue for study, and the lack of appropriate control tissue. We have therefore used a hapten labelled deoxyoligonucleotide cocktail to study VEGF mRNA expression by non-isotopic in situ hybridisation in groups of patients with different proteinuric glomerular lesions and rigorously matched controls. Our working hypothesis was that proteinuria results from abnormal VEGF expression which increases the permeability of the glomerular basement ultrafiltration membrane.

\section{Methods}

Renal biopsy tissue was derived from the archive stores at the department of pathology, Leicester General Hospital. Ten cases of minimal change and membranous nephropathy and eight cases of diabetic nephropathy were identified. An equal number of controls was identified from the same archives, matched for age, sex, race, and fixation and storage time. Biopsies from diagnostic groups were performed during investigation of the nephrotic syndrome. All individuals had normal excretory renal function. Controls were patients 
undergoing renal biopsy for isolated microscopic haematuria in whom subsequent histological, immunofluorescent, and electron microscopic analysis was normal. All biopsies were taken during the period March 1991 to July 1996. All the patients with minimal change nephropathy, membranous nephropathy, or diabetic nephropathy were heavily nephrotic, with urinary protein losses of between 2.5 and $21 \mathrm{~g} / 24$ hours.

PROBE AND PROBE LABELLING

Twelve deoxyoligonucleotides (Pathway Services) complementary to the VEGF mRNA were used. All deoxyoligonucleotides were 30 bases long; this length has been established as a practical compromise between hybrid stability, hybrid specificity, cost of synthesis and purification, and tissue penetration efficiency. ${ }^{8}$ Deoxyoligonucleotides were 3 ' and 5' labelled with 2,4-dinitrophenyl (DNP) during solid phase chemical synthesis (Oswel DNS Service). DNP labelling has been shown to produce stonger non-isotopic in situ hybridisation (ISH) signal than other hapten labels. ${ }^{9}$

NON-RADIOISOTOPIC ISH

The protocol for non-radioisotopic ISH was adapted from those we have previously reported. ${ }^{9}{ }^{10} \mathrm{RNase}$-free reagents and glassware (diethylpyrocarbonate (DEPC) treated; Sigma; D5758) were used throughout. Sections $(4 \mu \mathrm{m})$ were cut onto silane coated slides. Sections were dewaxed in xylene and taken through graded alcohols. Pretreatments included $0.2 \mathrm{M}$ $\mathrm{HCl}$ for eight minutes and proteinase $\mathrm{K}$ at $37^{\circ} \mathrm{C}$ for one hour. Various proteinase $\mathrm{K}$ concentrations were used (range $2-10 \mu \mathrm{g} / \mathrm{ml}$ ) to elicit the optimum digestion for each block. Slides were then post-fixed with $0.4 \%$ paraformaldehyde/phosphate buffered saline at $4^{\circ} \mathrm{C}$ for 10 minutes and acetylated with $0.25 \%$ acetic anhydride/xylene. After this the sections were covered for 10 minutes in prehybridisation buffer: $600 \mathrm{mM} \mathrm{NaCl}, 1 \times \mathrm{PE}, 10 \%$ dextran sulphate, $30 \%$ formamide. (PE $=50 \mathrm{mM}$ Tris ( $\mathrm{pH} 7.5$ ), $0.2 \%$ bovine serum albumin, $1 \%$ sodium dodecylsulphate, $1 \%$ polyvinylpyrrolidone (40 kDa), 1\% Ficoll (400 kDa.)

Fifteen microlitres of the labelled probe cocktail (250 ng probe $/ \mathrm{ml}$ prehybridisation buffer) were pipetted onto a coverslip, the section was drained of excess fluid, inverted, and allowed to pick up the coverslip. A two hour hybridisation at $37^{\circ} \mathrm{C}$ was performed. Posthybridisation washes were as follows: $2 \times 10$ minutes standard saline citrate solution (SSC)/ $30 \%$ formamide at $37^{\circ} \mathrm{C}, 2 \times 5$ minutes SSC at room temperature, blocking solution for 15 minutes. Sections were then incubated with alkaline phosphatase labelled polyclonal antiDNP $\left(\mathrm{Fab}_{2}\right)$ antibody (kindly donated by Dako, UK) 1:100 in blocking solution for 30 minutes. Sections were washed in blocking solution for five minutes and in buffer $3(0.1 \mathrm{M}$ tris- $\mathrm{HCl}, \mathrm{pH}$ 9.5, 0.1 M NaCl, 0.05 $\mathrm{M} \mathrm{MgCl}_{2}$ ) for five minutes. They were incubated overnight in NBT/BCIP developing substrate-44 $\mu \mathrm{l}$ of nitroblue tetrazoleum (NBT; Sigma; 75 $\mathrm{mg} / \mathrm{ml}$ in $70 \%$ dimethylformamide) plus $33 \mu \mathrm{l}$ of 5-bromo-4-chloro-3-indoyl phosphate (BCIP; Sigma: $50 \mathrm{mg} / \mathrm{ml}$ in dimethylformamide) in $10 \mathrm{ml}$ of buffer 3 . Finally slides were washed under running tap water, counterstained with haematoxylin, and mounted with an aqueous mountant.

PROBE SPECIFICITY

Specificity of this probe cocktail was confirmed by the appropriate use of ISH negative controls as previously listed. ${ }^{10}$ These were: probe omission, antibody omission, RNAse A1 pretreatment, random oligonucleotide similarly labelled, and non-homologous probe cocktail with the same G-C content as the VEGF probe cocktail (that is, $55 \%$ ).

ENUMERATION AND STATISTICS

VEGF mRNA positive cells were counted in all the glomeruli (range 11 to 36 ) in every biopsy by a single observer who was unaware of the nature (control or patient) of the specimen. The counting was performed at $\times 1000$ magnification after glomeruli had been identified at lower magnification. Preliminary experiments on samples not used in this study had shown that counting under oil immersion, although prolonged, was a robust and reliable method with $1-2 \%$ error rate on repeated enumeration. Cell counts were expressed per unit cross sectional diameter of the glomeruli (cells per 100 $\mu \mathrm{m}$ diameter) to allow for potential differences in glomerular size. Glomerular size was measured with an eyepiece graticule at the time of counting. A mean figure for each biopsy was therefore obtained.

The results were then compared by Student $t$ test and are expressed as mean (SEM). Linear regression analysis was used to analyse any potential relation between VEGF mRNA positive cell number and the degree of proteinuria.

\section{Results}

Non-isotopic ISH produced a crisp well defined signal in all cases, with negligible background. ISH negative controls showed no signal on any occasion. Morphology was well preserved. The site of VEGF mRNA expression was confirmed to be predominantly within the glomeruli, specifically the visceral glomerular epithelial cells (fig 1). Occasionally a positive signal was seen in tubular cells (fig 1A) but this was only identified in three of the 56 biopsy specimens.

More VEGF mRNA positive glomerular cells (per unit cross sectional diameter) were identified in the minimal change biopsies (mean (SEM), $19.35(1.5)$ ) than in the control samples (12.6 (1.73)), p $<0.01$ (fig 2). In addition there was a significant correlation between 24 hour protein excretion at the time of biopsy and the number of VEGF mRNA cells per glomerulus $(r=0.08, \mathrm{p}=0.01)$.

In contrast, significantly fewer VEGF mRNA positive cells were seen in biopsies from diabetic patients $(5.93(0.97))$ compared with controls (9.97 (1.25)), $\mathrm{p}<0.03$ (fig 2).

There was no difference in numbers of VEGF mRNA positive glomerular cells between the membranous nephropathy biopsies 

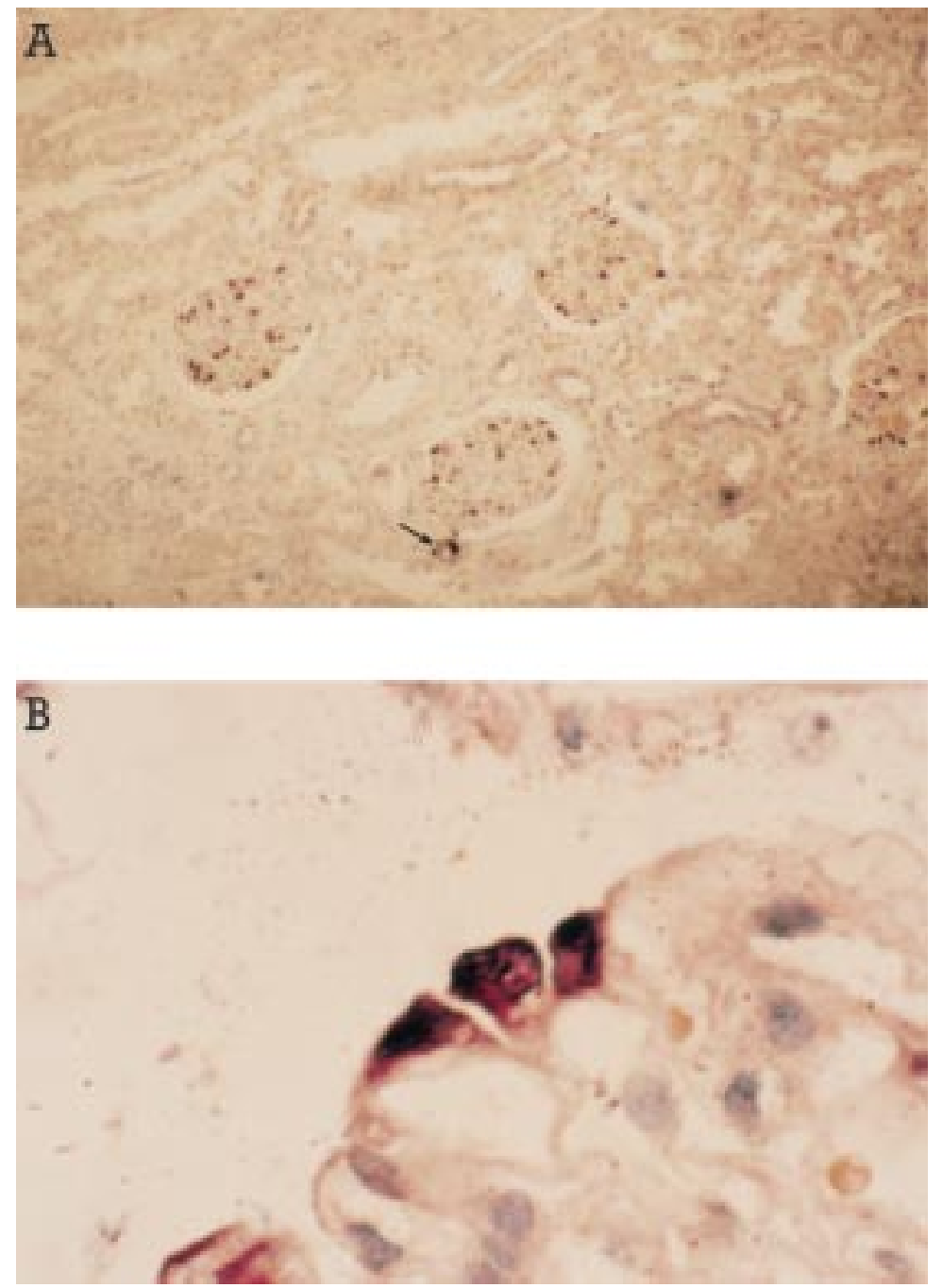

Figure 1 Non-isotopic in situ hybridisation for vascular endothelial growth factor (VEGF) $m R N A$. NBT/BCIP identification. Haematoxylin counterstain. $(A): \times 239$ (original magnification $\times 400$ ). Tubular cell expression uncommon (arrow). (B)

Predominant expression clearly within podocytes: $\times 598$ (original magnification $\times 1000$; oil immersion).

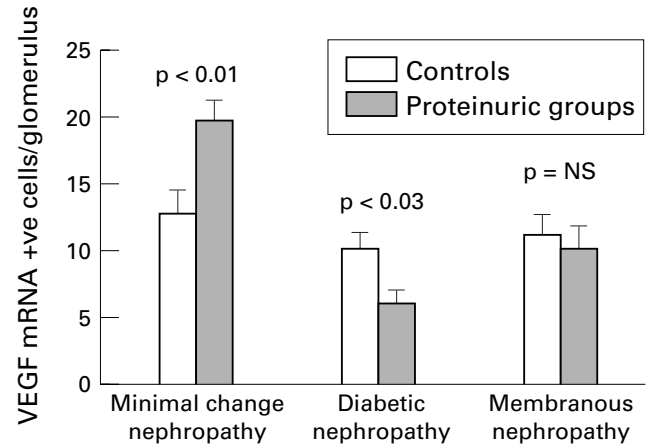

Figure 2 Vascular endothelial growth factor (VEGF) $m R N A$ positive glomerular cells/per unit cross sectional glomerular diameter in controls and patients with proteinuric glomerular lesions.

(10 (1.62)) and the controls (10.98 (1.51)), NS. No correlation was seen between cell numbers and 24 hour urinary protein loss.

\section{Discussion}

This is the first report to identify VEGF mRNA expression using non-isotopic in situ hybridisation. It is also the first study to exam- ine ISH VEGF mRNA expression within a reasonable number of cases from different diagnostic groups and to make comparisons with appropriately matched controls. Although previous ISH studies on normal (developing or adult $)^{23}$ and diseased kidney ${ }^{11}$ have been reported, these studies used isotopic ISH (which always has the weakness that signal may extend to adjacent cells from neighbouring positive ones), were performed on small numbers of specimens, or used unmatched controls.

In addressing the working hypotheses, our data are conflicting. The data from the minimal change experiment support the hypothesis, as we identified increased numbers of VEGF mRNA positive glomerular cells and the number may correlate with the degree of proteinuria. However, the data from the diabetic and membranous patients are contradictory: although these individuals were heavily nephrotic, the diabetic biopsies showed fewer VEGF mRNA positive cells than controls and the membranous biopsies similar numbers to controls. Although it would have been preferable to study other diagnostic groups (proteinuric proliferative glomerulonephritis, IgA disease, mesangiocapillary nephropathy, and so on), a review of our archives did not yield sufficient numbers in any of the other diagnostic groups with the necessary controls.

The minimal change biopsies, by definition, were normal on light microscopy, unlike the diabetic and membranous nephropathy samples. Indeed, since the natural history of these clinical disorders dictates that biopsies tend to be done when the disease process is well established (and may have been so for years), it was no surprise to identify a significant degree of glomerular sclerosis in these two groups. Clearly when normal glomerular architecture is disrupted, fewer viable cells (of all types) will remain. This may explain the reduced numbers in the diabetic biopsies, in which glomerular sclerosis was marked. However, these patients were still nephrotic. This suggests that even if abnormal VEGF biology were the sole pathogenic factor in minimal change disease, it is not the whole story. Other factors must contribute in other forms of glomerular disease.

VEGF is a complex molecule and has several different forms. Differential exon splicing of the VEGF gene results in three main mRNA species which code for three secreted isoforms: VEGF189, $\mathrm{VEGF}_{165}$, and $\mathrm{VEGF}_{121} \cdot{ }^{12-14}$ All three have been demonstrated in sieved glomeruli and unseparated infant and adult renal tissue. These isoforms are physicochemically and possibly functionally distinct. Our deoxyoligonucleotide cocktail was complementary to all VEGF isoforms. As the difference between isoforms results from exon deletion, almost all their sequences are identical, the only specific portion being the spliced exon boundaries. Only one oligonucleotide would be specific for this boundary sequence. Unfortunately standard non-isotopic ISH is not sensitive enough to work in these circumstances, despite the newer hapten labels. ${ }^{9}$ To 
add to the complexity, there are at least three receptors for VEGF. One of these, soluble-Flt, is a form which inactivates VEGF when bound.

In conclusion, non-radioisotopic ISH confirms that VEGF mRNA is almost exclusively expressed by visceral glomerular epithelial cells. Abnormal numbers of cells are seen in both minimal change disease and diabetic nephropathy. As VEGF and its receptors exist in several distinct isoforms, further study of qualitative VEGF isoform and receptor expression in diagnostic groups is indicated as future technology allows.

This work was supported by the National Kidney Research Fund, grant R32/2/96. SJH is supported by Wellcome Trust grant $057936 / \mathrm{Z} / 99 / \mathrm{Z}$

1 Williams JD, Coles GA. Proteinuria-a direct cause of renal morbidity. Kidney Int 1994;45:443-50.

2 Simon E, Gröne H-J, Johren O, et al. Expression of vascular endothelial growth factor and its receptors in human renal ontogenesis and in adult kidney. Am f Physiol 1995;268: 240-50.

3 Brown LF, Berse B, Tognazzi K, et al. Vascular permeability factor mRNA and protein expression in human kidney. Kidney Int 1992;42:1457-61.
4 Simon M, Röckl W, Hornig C, et al. Receptors of vascular Simon $M$, Röckl W, Hornig C, et al. Receptors of vascular
endothelial growth factor/vascular permeability factor VEGF/VPF) in fetal and adult human kidney: localisation and $\left.{ }_{125}^{125} \mathrm{I}\right]$ VEGF binding sites. F Am Soc Nephrol

5 Senger DR, Connelly DT, Van de Water L, et al. Purification and N-terminal amino acid sequence of guinea-pig tumorsecreted vascular permeability factor. Cancer Res 1990;50: 1774-8.

6 Roberts WG, Palade GE. Increased microvascular permeability and endothelial fenestration induced by vascular endothelial growth factor. F Cell Sci 1995;108:2369-79.

7 Gröne H-J. Angiogenesis and vascular endothelial growth factor (VEGF): is it relevant in renal patients. Nephrol Dial Transplant 1995:10:761-3.

8 Pringle JH, Ruprai AK, Primrose L, et al. In situ hybridisation of immunoglobulin light chain mRNA in paraffin sections using biotinylated or hapten-labelled oligonucleotide probes. F Pathol 1990;162:197-207.

9 Harper SJ, Bailey E, McKeen C, et al. A comparative study of digoxigenin, 2,4-dinitrophenyl and alkaline phosphatase of digoxigenin, 2,4-dinitrophenyl and alkaline phosphatase as deoxyoligonucleotide labels in non-isoto

10 Jones PH, Harper SJ, Watts F. Stem cell patterning and fate in human epidermis. Cell 1995;80:83-93.

11 Shulman K, Rosen S, Tognazzi K, et al. Expression of vascular permeability (VPF/VEGF) is altered in many glomerular diseases. F Am Soc Nephrol 1996;7:661-6.

12 Ferrara N, Davis-Smith T. The biology of vascular endothelial growth factor. Endocr Rev 1997;18:4-25.

13 Houck KA, Ferrara N, Winer J, et al. The vascular endothelial growth factor family: identification of a fourth molecuar species and characterization of alternative splicing of RNA. Mol Endocrinol 1991;5:1806-14.

14 Poltorak Z, Cohen T, Sivan R, et al. VEGF145, a secreted vascular endothelial growth factor isoform that binds to extracellular matrix. f Biol Chem 1997;272:7151-8. 